\title{
Restauración del Tajo de San Pedro en La Alhambra de Granada. Aspectos de cálculo
}

\section{The restoration of San Pedro cliff at La Alhambra. Calculation aspects}

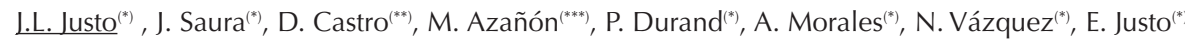

RESUMEN

La muralla-palacio de La Alhambra, que es Patrimonio de la Humanidad, se ve amenazada por el avance del Tajo de San Pedro (Figura1), un diedro de $65,5 \mathrm{~m}$ de altura, que corta la colina de La Alhambra y que se ha situado a sólo 23,8 $\mathrm{m}$ de ella.

Existe en la zona un régimen tectónico de extensión que afloja el terreno y activa la caída de lajas.

Otra causa importante del deterioro del Tajo es la erosión. El coeficiente de seguridad al deslizamiento del Tajo sometido al terremoto de periodo de retorno de 1.000 años es de 0,73.

La solución propuesta para su preservación es una malla de alambre postesada de alto límite elástico y revegetación autóctona.

La presión que puede llegar a aplicar la malla en servicio eleva el coeficiente de seguridad con sismo a un valor próximo a uno. Mediante una simulación en ordenador se ha analizado el impacto ambiental.

489-3

Palabras clave: muralla-palacio, lajas, coeficiente de seguridad, impacto ambiental.

\footnotetext{
(*) Universidad de Sevilla (Sevilla, España)

(*) Universidad de Cantabria (España)

${ }^{(* *)}$ Universidad de Granada (Granada, España)
}

Persona de contacto/Corresponding author: jlj@us.es (J. L. Justo)

\section{SUMMARY}

San Pedro cliff (Figure 1) is a dihedral $65.5 \mathrm{~m}$ high, which has progressed to place itself at $23.8 \mathrm{~m}$ from the Alhambra wall-palace that is a heritage of the Humankind.

Active normal faults surrounding the cliff have created an extension tectonic regime that loosens the ground and activates the slab falls.

One of the faces of the dihedral is a major fault. The cliff's factor of safety subject to the 1000 years return period earthquake is 0.73 .

The preservation solution proposed is a posttensioned high elastic limit wire mesh and autochthonous vegetation.

With this reinforcement, the factor of safety under that earthquake raises to a value around one. $A$ computer simulation has permitted to analyse the environmental impact.

Keywords: wall-palace, slabs, cliff's factor, environmental impact. 


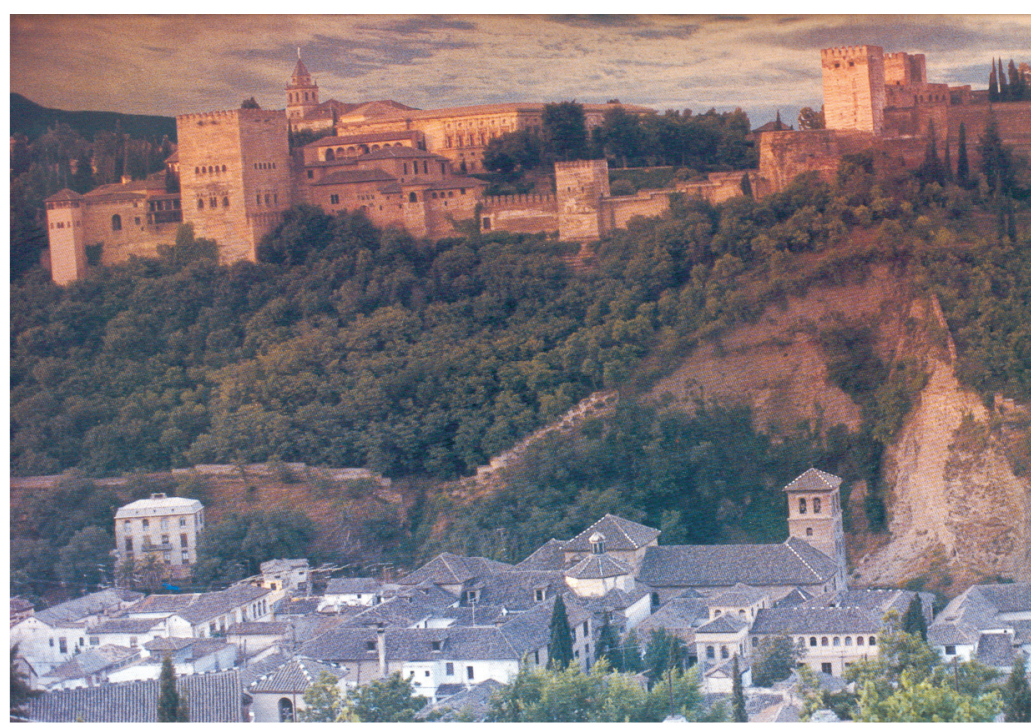

1. El Tajo de San Pedro bajo las murallas de La Alhambra, con el Darro a su pie y frente a la Iglesia de San Pedro y San Pablo.

\section{INTRODUCCIÓN}

Las ciudades y pueblos colgados, construidos en el borde de un acantilado y sometidas a frecuentes deslizamientos, ocupan un lugar significativo dentro de los problemas planteados en la preservación de sitios históricos. Dentro de estos pueblos colgados con problemas se encuentran Arcos de la Frontera, Benamejí y Carmona. Estos corrimientos han sido corregidos hasta ahora con métodos caros y no siempre eficientes.

Un exponente de este problema es el Tajo de San Pedro (Figura 1), un diedro de 65,5 m de altura, cuya proximidad a la muralla-palacio de La Alhambra, que es Patrimonio de la Humanidad, hace temer por la integridad de ésta. Los desprendimientos del terreno han destruido ya una parte importante de la cerca cristiana, construida en 1526, y cuyo valor arqueológico es importante.

\section{ANTECEDENTES}

Un excelente estudio sobre los antecedentes del Tajo de San Pedro ha sido realizado por José María Rodríguez Ortiz (1), un resumen del cual, matizado por los autores de este texto, se expone a continuación.

El Tajo de San Pedro debió estar presente en la morfología del Cerro de la Alhambra desde mucho antes de la construcción del Conjunto Monumental ya que se trata de un accidente natural creado por la interacción del Darro, la tectónica y la erosión desde la época en que dicho río se encajó en sus proximidades, probablemente desplazado por inestabilidades de la ladera opuesta.

El peligro para la cerca o muralla debía ser importante desde la construcción del palacio, y existen referencias de que en 1520 se había realizado un relleno de tierras al pie del escarpe como espaldón de protección. Es la enorme terrera o presa que aparece en algunos textos y que correspondería a una gran mota o malecón lateral que separaría el río del pie del escarpe.

Tras el incendio de 1524 se dice: “... poco tiempo después de este desgraciado suceso comenzaron los desprendimientos de terrenos en lo que hoy se llama el Tajo de San Pedro, lo que causó notables desperfectos en la Alcazaba y aún en los restos del Cuarto Dorado". Podría inferirse que el incendio acabó con la vegetación del Cerro, dejando el terreno desprotegido y desprovisto del armado que prestaban sus raíces, por lo que pudo favorecerse la inestabilidad. Además, parece indicar que se había dispuesto un aliviadero de los aljibes sobre la citada terrera, que pudo contribuir a la destrucción de la misma.

El estudio en profundidad de un grabado hecho en 1564 (Figura 2) muestra que en ese momento la cuña estaba a una distancia en horizontal de unos $60 \mathrm{~m}$ de la muralla de La Alhambra y que la altura total del Tajo era de 33 m. Una comparación con la Figura 1 indica el importante avance de la erosión del Tajo en algo más de cuatro siglos. En 1600 una crecida del río socava la base de la colina de La Alhambra y deja al Tajo en una situación que empieza a parecerse a la actual, según puede verse en un grabado del siglo XVII. Los desprendimientos de 1600 y 1601 provocan, además, el abatimiento de la parte superior de la cerca.

"Hubo enfrentamientos entre los Alcaides de la Alhambra y los Caballeros Veinticuatro, del Cabildo de la Ciudad, por los desprendimientos de 1600 y 1601 . Además de la conocida acción erosiva del Darro se citan los posibles recalos y reblandecimientos de la Acequia de Romaila o de Santa Ana que bajo dicho cerro llevaba y lleva en la actualidad el agua para el suministro de las casas de la ciudad'.

La parte superior de la cerca desaparece en el plano general de la Alhambra de Hermosilla y Villanueva de 1804, en mucha mayor profusión que en el de 1775, lo que parece indicar, siguiendo a Rodríguez Ortíz, que se produjo una clara rotura de dicha parte superior en el periodo 1788-1804. En los planos de 1812 y 1815 1a rotura de la cerca es semejante.

\section{GEOLOGÍA}

El palacio de La Alhambra está construido en la cima de una colina, cuyo pie se encuentra rodeado por el río Darro en su lado norte (Figura 1). 
El Tajo de San Pedro corta una serie de niveles de conglomerados densos que constituyen la llamada formación Alhambra, de edad Plioceno inferior a Pleistoceno, y corresponden a abanicos aluviales procedentes de la erosión de Sierra Nevada. Las piedras son redondeadas, con un tamaño medio de $10 \mathrm{~cm}$. La matriz $<0,08 \mathrm{~mm}$ oscila entre un 13 y un $35 \%$ y es normalmente arena limosa, a veces arcillosa. Hay también capas, de $1 \mathrm{~m}$ de espesor, de arcilla limosa.

\section{TECTÓNICA}

Varias fallas normales activas, con dirección NO-SE se localizan en los alrededores de La Alhambra (Figura 3).

Un régimen tectónico de distensión ha creado estas fallas. En la actualidad una serie de fallas

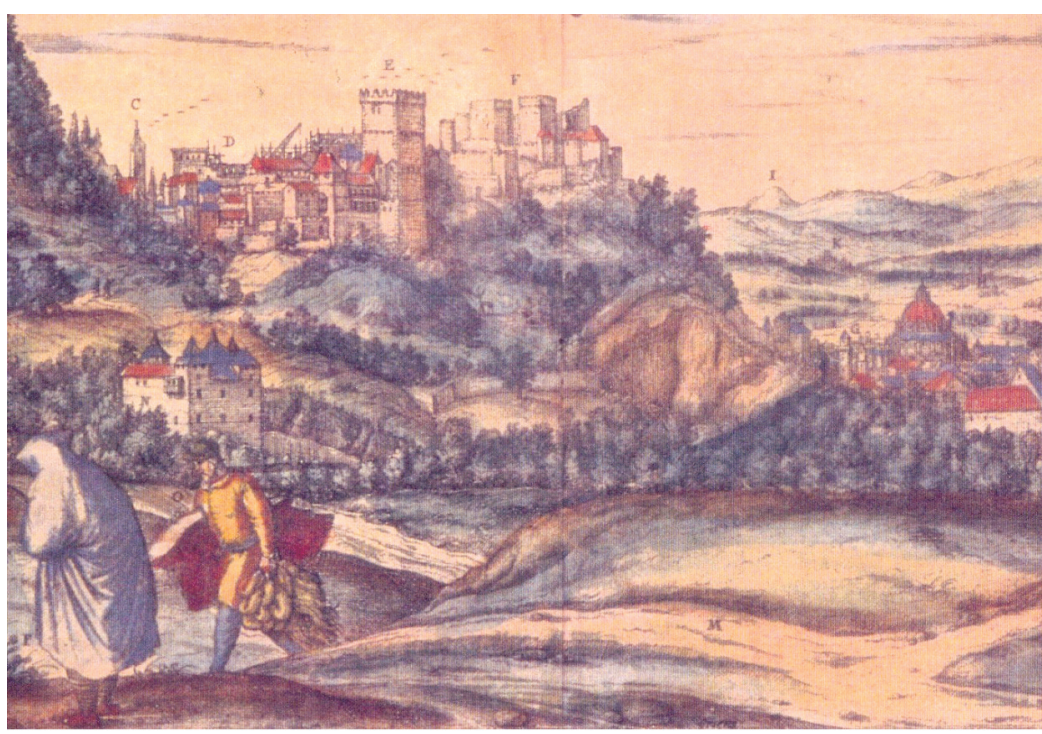
normales activas cruza el Tajo de San Pedro.

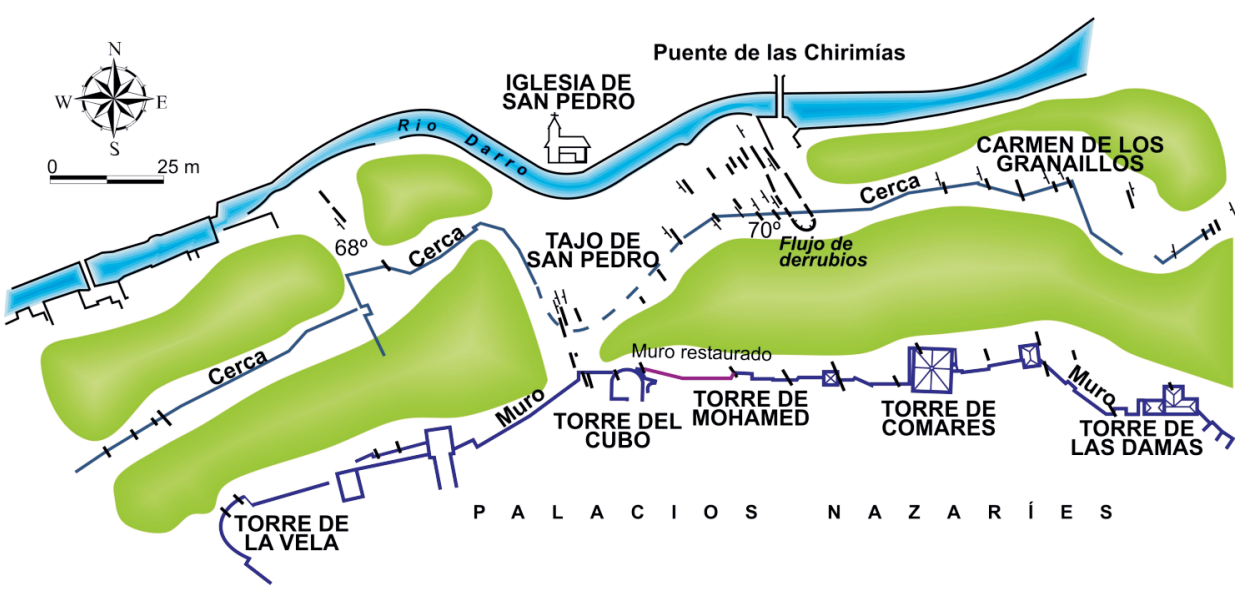

Una de estas fallas, cuya dirección es N $158^{\circ}$ constituye la cara oeste del diedro (Figura 1). El salto de falla de la cara Este es de $7 \mathrm{~m}$. La Figura 4a muestra el esquema de funcionamiento de una falla normal: la reducción de las tensiones horizontales producida por el régimen tectónico de extensión puede conducir a la rotura en el volumen focal.

El buzamiento medio de esta falla $(\alpha)$ es de $70^{\circ}$, y esto nos da la mejor estimación del ángulo de rozamiento interno. Suponiendo que el plano de falla coincida con el plano de contacto del círculo de Mohr con la envolvente, de la figura $4 \mathrm{~b}$ se deduce que:

$\Phi^{\prime}=2 \alpha-90^{\circ}=50^{\circ}$

\section{RECONOCIMIENTO DEL TERRENO}

El Centro de Estudios y Experimentación de Obras Públicas realizó varios sondeos en la zona del Tajo de San Pedro (2). Una investigación más profunda fue Ilevada a cabo por Geocisa (3), como parte del

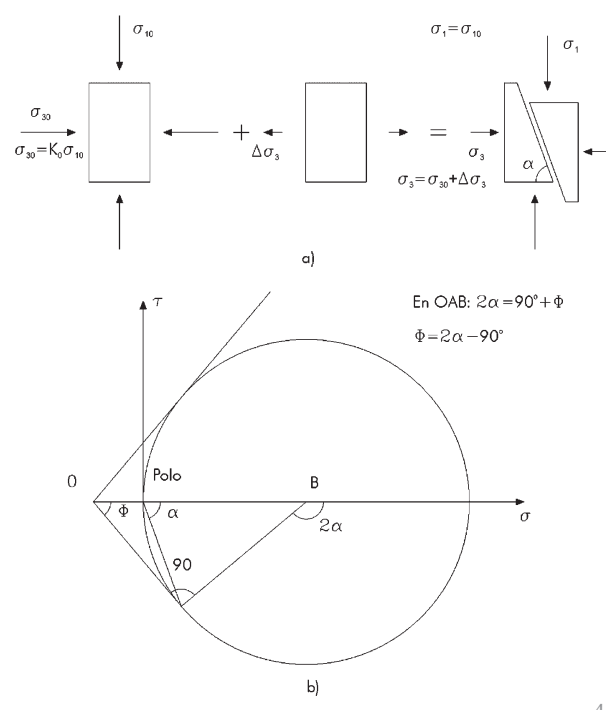

2. Grabado de Hüfnagel de 1564 (cortesía de J. Calatrava)

3. Esquema del recinto de La Alhambra y las zonas próximas, en la que se han representado las principales fallas. Obsérvese el meandro que forma el río Darro a la altura del Tajo.

4. Tensiones actuantes en una falla normal. 
ensayos de permeabilidad, de penetración, presiométricos, down-hole y cross-hole y ensayos de laboratorio. Los ensayos de penetración dieron siempre rechazo.

De arriba abajo se pueden distinguir las siguientes capas de suelo en la formación Alhambra:

1. Conglomerado 1, denso. Tamaño máximo $10 \mathrm{~cm}$. Recuperación de testigo 100\%.

2. Conglomerado 2, muy denso. Tamaño máximo 5-8 cm. Recuperación de testigo $100 \%$.

3. Conglomerado 3, densidad moderada. Recuperación de testigo $60 \%$.

4. Capa 4. Derrubios en el pie del talud. Bloques, gravas y arenas sueltas de naturaleza cuarcítica y filítica, con mayor proporción de la fracción de arena.

5. Conglomerado 5, de arena y grava, con elevada cantidad de finos limosos. Densidad muy alta, permeabilidad muy variable.

5 bis. Capitas de $1 \mathrm{~m}$ de arcilla dura intercaladas en la capa 5. Recuperación 100\%.

La Tabla 1 presenta un resumen de los ensayos más importantes efectuados sobre las distintas capas.

\section{RESISTENCIA AL CORTE DEL CONGLOMERADO}

En una búsqueda bibliográfica exhaustiva, sólo se ha encontrado un caso en el cual se ha medido la resistencia de un conglomerado en un ensayo de corte directo in situ (5). Este conglomerado pertenecía al Terciario y dio un ángulo de rozamiento interno de $62^{\circ}$, con cohesión nula. Tampoco se han encontrado estimaciones válidas de la resistencia de conglomerados.

El Centro de Estudios Ménard (6) recomienda una relación entre la presión límite y el ángulo de rozamiento interno, de la que se deduce la siguiente ecuación:

$\Phi^{\prime}=13,288 \log p_{1}{ }^{*}-7,86$

siendo $p_{1}^{*}$ la presión límite neta en kPa.

Con los valores de la presión límite de la Tabla 1 , se obtienen los valores de $\Phi^{\prime}$ que figuran en la columna siguiente. Baguelin et al. (7), citan la fórmula del Centro de Estudios Ménard, pero señalan que "actualmente no existen procedimientos teóricos para convertir los ensayos del presiómetro en parámetros efectivos del suelo $C^{\prime}$ y $\Phi^{\prime}$.

Sin embargo, la comparación de las estimaciones de Ménard con los resultados de ensayos triaxiales y de corte directo en arena, que presentan estos autores, da una concordancia bastante buena, aunque a veces deja algo del lado de la seguridad.

En el Tajo de San Pedro sólo se pudieron hacer ensayos de compresión simple y de corte directo con drenaje en la capa 5 bis (Tabla 1). En el ensayo de corte directo con drenaje se satura la muestra en el laboratorio, mientras que en el terreno la muestra sólo se debe saturar superficialmente en épocas de lluvia intensa. La resistencia a compresión simple está ligada con los parámetros de Coulomb por medio de la ecuación:

$C=\frac{q_{u}}{2} \frac{1-\operatorname{sen} \Phi}{\cos \Phi}$

Esta ecuación nos daría, para $q_{\mathrm{u}}=394 \mathrm{kPa}$ y $\Phi^{\prime}=31^{\circ}$ (véase Tabla 1 ), una cohesión para el material no saturado de $111 \mathrm{kPa}$.

En las dos últimas columnas se indican los parámetros escogidos para el cálculo. Se reconoce que esta elección es discutible, y está basada en los siguientes argumentos:

1. Se ha dado bastante peso a la relación entre el buzamiento de la falla y el ángulo de rozamiento interno (apartado 4). Este ángulo de rozamiento interno $\left(50^{\circ}\right)$ es, además, intermedio entre los correspondientes al ensayo de corte directo y a las estimaciones en función del ensayo presiométrico que se indican en el apartado 6. Se ha supuesto que un límite inferior de la cohesión de las capas 1 y 2, es el valor de $c^{\prime}$ obtenido en la capa 5 bis (32 kPa).

2. La cohesión se ha reducido a $18 \mathrm{kPa}$ en la capa 3, donde los parámetros presiométricos son inferiores, y se ha aumentado en la capa 5, donde estos parámetros y los geofísicos son superiores. Por el contrario, en esta última capa se ha reducido el ángulo de rozamiento interno a $45^{\circ}$, porque posee una elevada cantidad de finos limosos.

3. La superficie pésima de deslizamiento no atraviesa la capa 4, por lo que los parámetros escogidos para ésta (véase pie de la Tabla1) son poco importantes

\section{EVOLUCIÓN DEL TAJO DE SAN PEDRO}

El Tajo de San Pedro es el resultado de las riadas del río Darro, la tectónica, la erosión y tal vez las filtraciones procedentes del palacio de La Alhambra. El ataque de la ladera de la colina de La Alhambra por el río durante las crecidas ha sido favorecido por las fracturas producidas en ella por el régimen tectónico de extensión. Estas riadas han creado un meandro convexo hacia el Tajo (Figura 3). Poco después de 1524 comienzan las noticias sobre los deslizamientos en el Tajo, cuando un fuego destruyó la vegetación de 


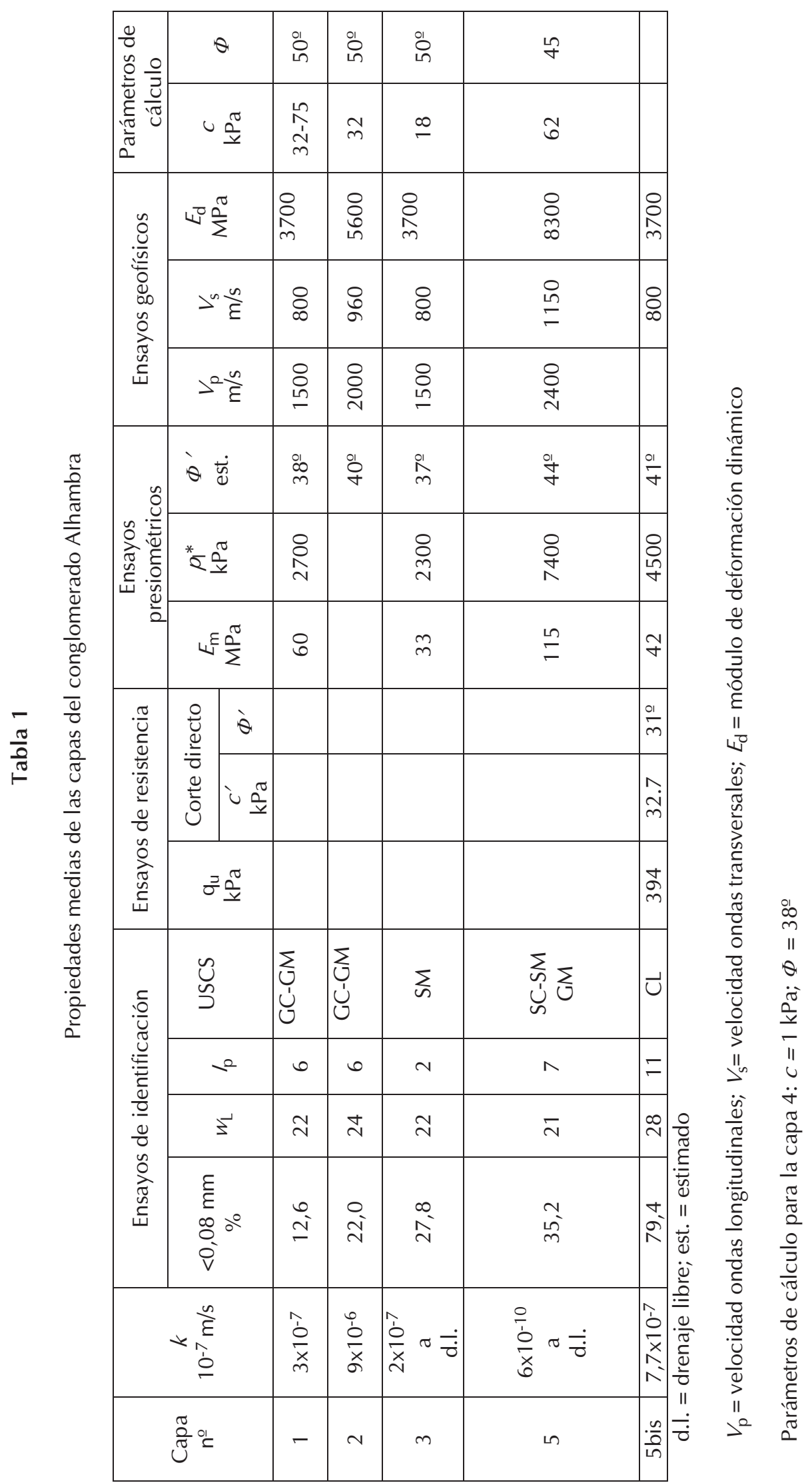

la colina de La Alhambra, dejando el suelo sin protección. Después de esta fecha, hay al menos siete desprendimientos bien documentados. Las causas citadas no son ya las crecidas del río, sino el agua vertida por los depósitos de La Alhambra, explosiones y filtraciones. El último desprendimiento de una laja sub-vertical en la cara de la falla, que afectó a un $30 \%$ de su extensión, se produce en 1985. 
De una comparación de los grabados y planos topográficos correspondientes a diferentes fechas se deduce que el desplazamiento de retroceso medio del borde puede ser de $8 \mathrm{~cm} /$ año.

\section{SITUACIÓN ACTUAL}

El régimen de extensión de la Neotectónica produce una reducción de las fuerzas horizontales del Tajo, que puede alcanzar el valor cero cerca de la superficie. Las juntas abiertas en el Tajo son ahora un camino preferente para las filtraciones que llegan desde el palacio. La altura del Tajo sobre el río es de 65,5 $\mathrm{m}$ en la actualidad y la distancia horizontal desde el borde del diedro hasta la muralla es hoy de $23,8 \mathrm{~m}$.

El tramo abrupto superior del talud tiene una altura de 45,5 m con una pendiente de $67,4^{\circ}$. Existen derrubios en el pie (capa 4 en el apartado 5).

El retroceso medio horizontal del borde del

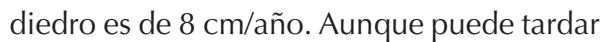
muchos años en llegar a la muralla, la historia muestra que la velocidad del proceso no es constante y puede ser acelerada por un terremoto importante, como se demostrará más adelante.

\section{SOLUCIONES PREVIAS}

El peligro que implica el progreso de la cuña de la muralla de La Alhambra ha sido previsto desde hace mucho, y desde 1520, las siguientes soluciones han sido propuestas y en algunos casos ejecutadas:

a) Terraplenes o muros en el pie del Tajo, para protegerlo de las crecidas del río.

b) Prohibición del riego del bosque de La Alhambra.

c) Desvío del río.

d) Un muro de tierra armada, de 9,2 m de altura, y una malla de triple torsión por encima, para favorecer el crecimiento de vegetación autóctona.

e) Un muro ecológico combinado con drenes californianos, un cosido de la ladera, micropilotes de refuerzo y tratamiento acrílico de la superficie del talud para evitar la erosión (1).

f) Inyección a través de una serie de tubos de acero sub-paralelos a la superficie del talud, y además regulación del río.

\section{REVISIÓN CRÍTICA DE LAS SOLUCIONES PREVIAS}

Según las instrucciones recibidas por parte del Patronato de la Alhambra, la solución adoptada debería tener un impacto medioambiental mínimo, lo que implica una intervención mínima.
El coste también debería ser mínimo. A pesar de su peligrosidad para la muralla de La Alhambra, el Tajo de San Pedro ha pasado a ser un elemento positivo en el paisaje urbano de la carrera del Darro, debido a su singularidad. La inclinación de la Torre de Pisa es el elemento que ha atraído a millones de visitantes, y, a pesar de que existía un peligro para la integridad de la torre, en ningún momento el municipio de Pisa se plantó enderezarla, sino sólo evitar que aumentara su inclinación.

En el caso del Tajo las soluciones no deben tratar de rellenar lo que ha hecho la naturaleza durante siglos, sino sólo estabilizarlo en su situación actual. Por ello se pueden hacer los siguientes comentarios respecto a las soluciones propuestas con anterioridad:

a) No es necesario ni conveniente construir nuevos terraplenes porque, como se dijo antes, el problema de las riadas no es importante ahora y en cualquier caso debería ser resuelto mediante la regulación del Darro río aguas arriba. En cualquier caso existe ya un pedraplén en el pie.

b) Para evitar que se marchite el bosque de La Alhambra es preciso regarlo.

c) El impacto de la desviación del río Darro sería inaceptable ya que es un elemento fundamental del paisaje urbano en un punto tan bello como la Carrera del Darro.

d) El impacto ambiental de una malla de triple torsión y de la tierra armada es también importante, ya que cambia drásticamente el escenario actual.

e) Esta solución, que resolvería el problema mecánico, fue rechazada por el Patronato de la Alhambra porque ocultaría un fenómeno geológico singular, como es el Tajo de San Pedro. El aspecto de las tuberías para recoger las aguas procedentes de los drenes californianos no sería atractivo y el tratamiento acrílico, que requeriría la demolición de las porciones sueltas del talud, impediría el crecimiento de la vegetación.

f) Como señala Rodríguez Ortiz (1), la inyección del conglomerado no está garantizada, debido al bajo coeficiente medio de permeabilidad del conglomerado, y, además, introduciría presión en planos subparalelos a la ladera lo que podría producir la caída de lajas del talud.

\section{EL USO DE MALLAS DE ALAMBRE EN LA PROTECCIÓN DE TALUDES}

La utilización de mallas de alambre ancladas para proteger laderas $(8,9)$ es un método barato y quizás el único posible para taludes altos y empinados.

Hay principalmente dos tipos: redes de cable y mallas de alambre de alto límite elástico. 


\subsection{Redes de cables (Figura 5)}

Torres Vila (8) ha presentado la cronología que sigue sobre este sistema de protección de taludes.

La utilización de redes de cable de acero en estabilidad de taludes se inicia en Suiza en la década que comienza en 1980, con el sistema Pentfix, desarrollándose una intensa labor de investigación en España y en Suiza a partir de la década que comienza en 1990. En 1993 se realiza la primera aplicación en España en el Puerto de Guetaria. Se trata de redes simplemente colgadas, sin bases de cálculo ni diseño.

A partir de esta fecha se desarrollan los primeros sistemas: CCA y CCS para rocas, BBS para suelos. En realidad el sistema se ha utilizado en todo tipo de terrenos duros. Para el diseño de la red se comienzan a ensayar los materiales en laboratorio, se inicia una cierta estandardización y se realizan cálculos aproximados de la estimación del soporte proporcionado por la red. Se trata todavía de sistemas pasivos.

En 1995 se realiza la primera aplicación en España de la red de cables como sistema de soporte, en el Puerto del Musel, en Gijón. En 1997 se realiza el primer sistema con anclajes activos, en la N-629, Ramales-Resines, en Cantabria, y se desarrollan los manuales de diseño.

Entre las realizaciones importantes destaca el desmonte de San Antolín (10), donde se utiliza una red de cables TECCO, para estabilizar los $60 \mathrm{~m}$ inferiores (pendiente del talud $40-55^{\circ}$ ) de un desmonte de $150 \mathrm{~m}$. Se trata de una zona de falla milonitizada.

Las redes de cable tienen los siguientes elementos:

a) Una malla de triple torsión con una abertura de $8 \times 10 / 15 \mathrm{~mm}$ y un alambre de diámetro superior a $2 \mathrm{~mm}$, para cerrar los espacios entre los cables de la red.

b) Una red de cables de acero de alta resistencia (1770 MPa) con abertura de 150 a 300 $\mathrm{mm}$ y espesor $8 \mathrm{~mm}$, para aplicar la presión al terreno.

c) Cables de acero de 16 a $24 \mathrm{~mm}$ de espesor y de 2 a 4 m de separación, que aplican una presión de 10 a $20 \mathrm{kPa}$ a la red de cables, a través de anclajes postesados en las intersecciones.

d) Anclajes internos y perimetrales postesados entre 100 y $500 \mathrm{kN}$.

Esta solución sería posible, pero la malla de acero de triple torsión es difícil de integrar con la vegetación del Tajo, debido a su pequeña abertura.

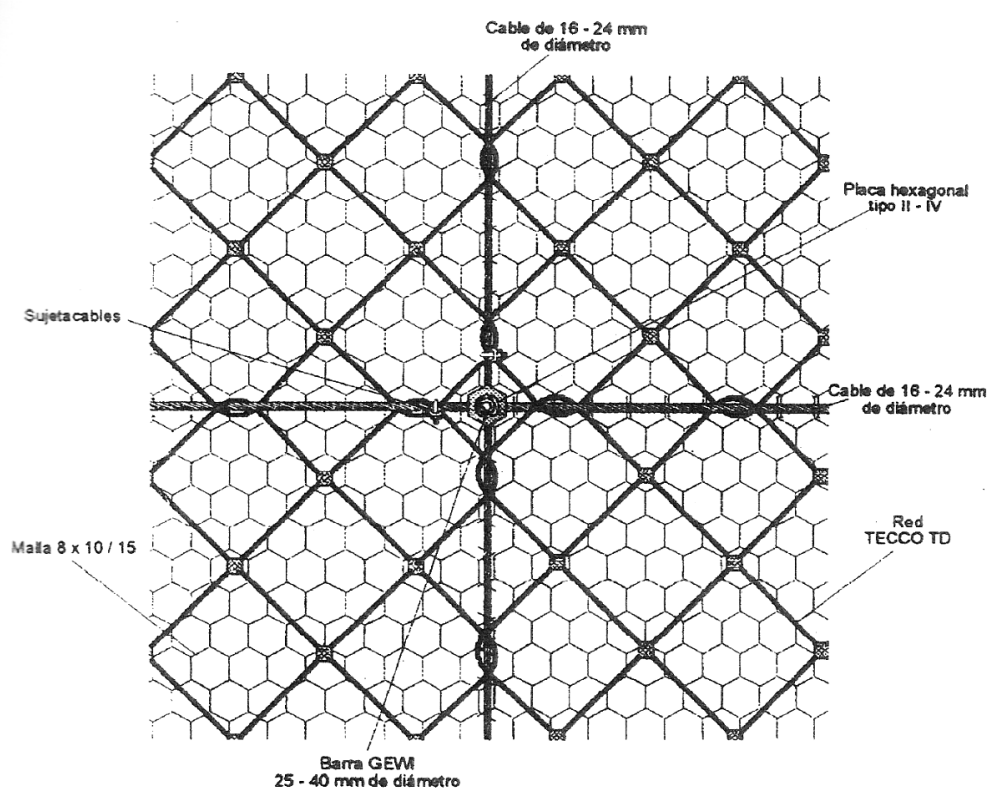

\subsection{Mallas de alambre de alto límite elástico (Figura 6)}

5. Red de cables (cortesía de Geobrugg)

Se desarrollan en la segunda mitad de los años 90, con el sistema TECCO, desarrollándose una amplia investigación sobre los materiales, destacando en este aspecto los ensayos realizados en el Laboratorio de Estructuras de la Universidad de Cantabria $(11,12)$.

Es una malla romboidal, con límite elástico entre 1770 y $2020 \mathrm{MPa}$ y un diámetro del alambre entre 3 y $4 \mathrm{~mm}$, colocada directamente sobre el talud con una abertura de 65 mm. La elección del diámetro puede depende de factores tales como la tensión a la que se vaya a someter la malla, el impacto visual o la probabilidad de corrosión en función de la meteorología. La presión sobre la superficie del talud (10 a $30 \mathrm{kPa}$ ) es aplicada por anclajes postesados aislados o reforzados por cables. Los anclajes pueden ser barras GEWI de espesor entre 25 y $40 \mathrm{~mm}$. En el perímetro de la zona tratada se colocan cables anclados de 20 a $24 \mathrm{~mm}$ de espesor. El diámetro de los anclajes depende de su tensión y de la probabilidad de corrosión según las características del terreno y la posición del nivel freático. La abertura de malla, de $65 \mathrm{~mm}$, es suficientemente pequeña para evitar la erosión del conglomerado y para mantener un impacto visual aceptable, especialmente si la vegetación actual se mantiene (al menos los árboles y arbustos) y se añaden nuevas plantas autóctonas.

La calle está a $40 \mathrm{~m}$ del Tajo, y la iglesia de San Pedro y San Pablo se encuentra en la línea de visión, por lo que es difícil distinguir la malla. Debido a todo eso, ésta fue la solución adoptada. 


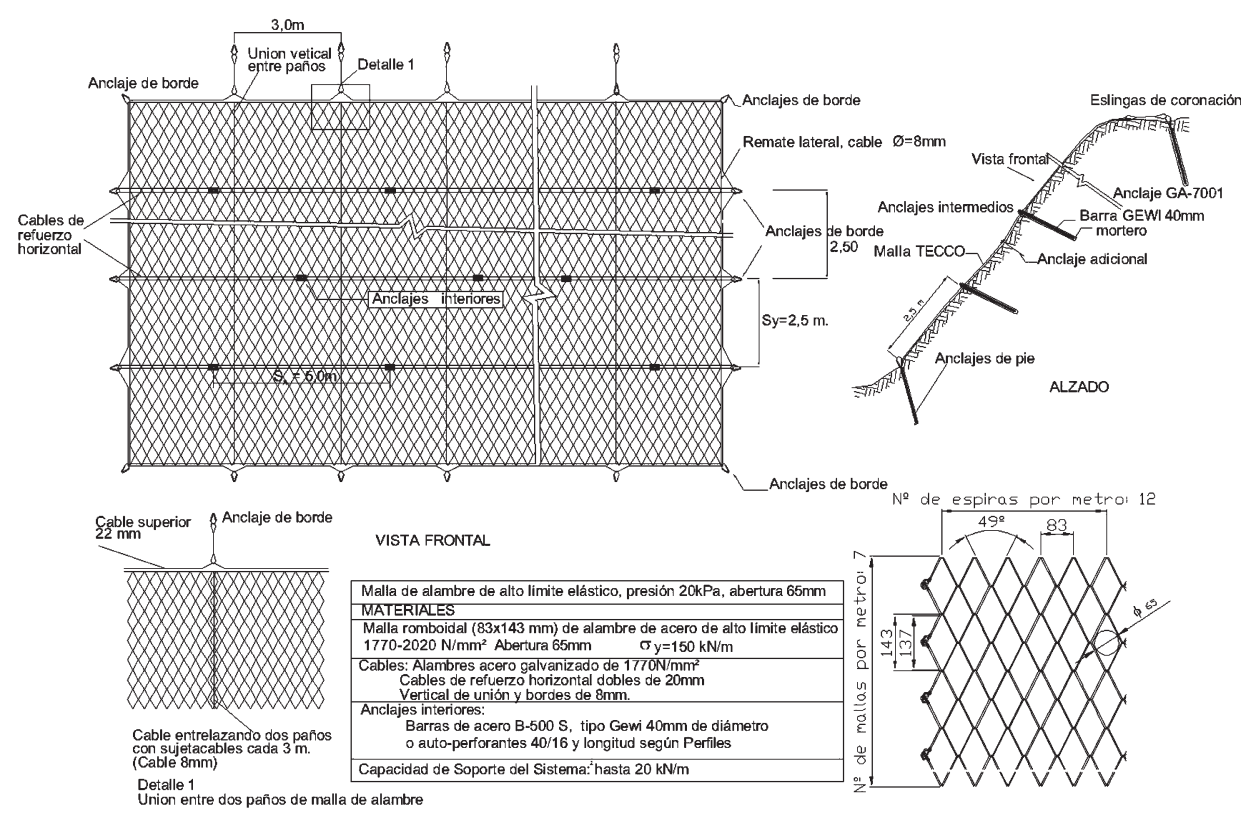

6. Malla de acero de alto límite elástico.
La secuencia constructiva es la siguiente (Figura 6):

1. Colocación de los anclajes, las eslingas y el cable de coronación.

2. Sujeta a este cable se extiende hacia abajo cada paño de malla, de unos $3 \mathrm{~m}$ de anchura.

3. Cada dos paños son entrelazados por un cable, que sigue la pendiente, de $8 \mathrm{~mm}$.

4. Se colocan los anclajes y cables de borde.

5. Se colocan, en su caso, los cables horizontales. Se tensan mediante los anclajes laterales de cable.

5. Se colocan y tensan los anclajes interiores, con la tensión que se deduce del cálculo.

Merece la pena destacar el escaso número de artículos publicados, fuera de España, sobre sistemas de protección flexibles $(13,14)$.

\section{CÁlCULO DE LAS MALLAS DE ALAMBRE DE ALTO LÍMITE ELÁSTICO}

\subsection{Parámetros de cálculo de la malla}

Torres Vila et al. (11) y Castro y Ballester (12) han sometido sectores de malla a tracción directa en la dirección de la diagonal mayor del rombo, con coacciones que impiden el movimiento lateral (deformación plana). En algunos ensayos se han medido también las tensiones laterales. Con ello se pueden obtener el módulo de elasticidad confinado, $E_{y c}$ (en realidad módulo de deformación), que es el que se va a utilizar en el modelo de deformación plana utilizado posteriormente, y el coeficiente de Poisson, $v$. Estos resultados se muestran en la Tabla 2.

\subsection{Modelo de deformación plana}

Torres Vila et al. (15) suponen que la rigidez de los cables de refuerzo horizontal es suficientemente grande como para admitir un modelo de deformación plana como el que se indica en la Figura 7. Se supone que la deformada de la malla al aplicar la carga del anclaje es un cilindro circular. Existen dos métodos de cálculo, basados ambos en la forma de la geometría impuesta y en la ley de Hooke, que se van a indicar a continuación.

El primero consiste en partir de la tensión de trabajo, $\sigma_{y}$ y del módulo de deformación $E_{y c}$ (v. Tabla 1) y encontrar la flecha del arco, $\Delta Z$, la capacidad de soporte unitario, $p$, y la carga de trabajo del anclaje, $Q$. El ángulo en el centro $\theta$, la cuerda, $S_{y}$ y el arco, $A$, están ligados por la ecuación (v. Torres Vila et al. (14)):

$\theta / 2 \approx\left[6\left(1-S_{y} / A\right)\right]^{1 / 2}$

La deformación unitaria de la malla es el incremento de deformación dividida por la longitud inicial:

$\varepsilon_{\mathrm{y}}=\left(A-S_{y}\right) / S_{y}$

Sustituyendo en [4] y operando:

$\theta / 2 \approx\left[6 \varepsilon_{y} /\left(1+\varepsilon_{y}\right)\right]^{1 / 2}$

La deformación unitaria en la dirección principal de deformación vale:

$\varepsilon_{y}=\sigma_{y} / E_{y c}$

La flecha del arco, $\Delta Z$, vale:

$\Delta Z=R[1-\cos (\theta / 2)]$ 
Tabla 2

Ensayos de tracción en malla Tecco G - 65

\begin{tabular}{|c|c|c|c|c|}
\hline $\begin{array}{c}\text { Espesor } \\
(\mathrm{mm})\end{array}$ & $\begin{array}{c}\sigma_{y r} \\
(\mathrm{kN} / \mathrm{m})\end{array}$ & $\begin{array}{c}\sigma_{y}=\sigma_{y r} \wedge, 67 \\
(\mathrm{kN} / \mathrm{m})\end{array}$ & $\begin{array}{c}E_{y c} \\
\mathrm{kN} / \mathrm{m}\end{array}$ & $v$ \\
\hline 3 & 151 & $\approx 90$ & $\approx 1898$ & 0,217 \\
\hline 4 & 260 & $\approx 155$ & $\approx 6250$ & 0,213 \\
\hline
\end{tabular}

$\sigma_{y r}=$ tensión de rotura de la malla

$\sigma_{y}=$ carga de trabajo en la dirección principal. *Para $\mathrm{F}=1,67$

$E_{y c}=$ módulo de deformación confinado

pero:

$R=S_{y} /[2 \operatorname{sen}(\theta / 2)]$

Sustituyendo en [8]:

$\Delta Z=\left(S_{y} /[2 \operatorname{sen}(\theta / 2)]\right)[1-\cos (\theta / 2)]$

Desarrollando en serie y simplificando:

$\Delta Z=S_{y} \theta(\mathrm{rad}) / 8$

El valor de $\theta$ se toma de la ecuación [6].

El equilibrio de fuerzas perpendiculares al talud a lo largo del arco (Figura 7) nos da la capacidad de soporte unitaria de la malla:

$$
\begin{aligned}
& p \int_{-\theta / 2}^{\theta / 2} p \cdot R \cdot d \alpha \cdot \cos \alpha=2 \sigma_{y} \cdot \operatorname{sen} \frac{\theta}{2} \\
& p \int_{-\theta / 2}^{\theta / 2} d S_{y}=2 \sigma_{y} \cdot \operatorname{sen} \frac{\theta}{2} \\
& p S_{y}=2 \sigma_{y} \cdot \operatorname{sen} \frac{\theta}{2}
\end{aligned}
$$

Sustituyendo [9] en [14]:

$p=\sigma_{y} / R$

La carga de trabajo del anclaje será:

$$
Q=p S_{x} S_{y}
$$

El segundo método consiste en partir de la capacidad de soporte unitaria deseada, $p, y$ hallar la flecha del arco, $\Delta Z$, y la tensión de trabajo, $\sigma_{y}$ que debe ser admisible y la carga de trabajo del anclaje.

$$
\Delta Z=\frac{\left(24 \mathrm{pS}_{\mathrm{y}}{ }^{4}\right)^{1 / 3}}{\left[\left(64 \mathrm{E}_{\mathrm{yc}}{ }^{2 / 3}-\left(96 \mathrm{pS}_{\mathrm{y}}\right)^{2 / 3}\right)\right]^{1 / 2}} \cong 0,36\left(\frac{\mathrm{pS}_{\mathrm{y}}{ }^{4}}{\mathrm{E}_{\mathrm{yc}}}\right)_{[15]}^{1 / 3}
$$

El arco vale:

$A=R \theta(\mathrm{rad})$

$R$ viene dado por la ecuación [9].

La suma de los ángulos interiores en el triángulo $\mathrm{OAB}$ tiene que sumar $\pi$. De ello se deduce:

$\theta(\mathrm{rad})=2 \pi-4 \operatorname{arctg} \frac{S_{y}}{2 \Delta Z}$

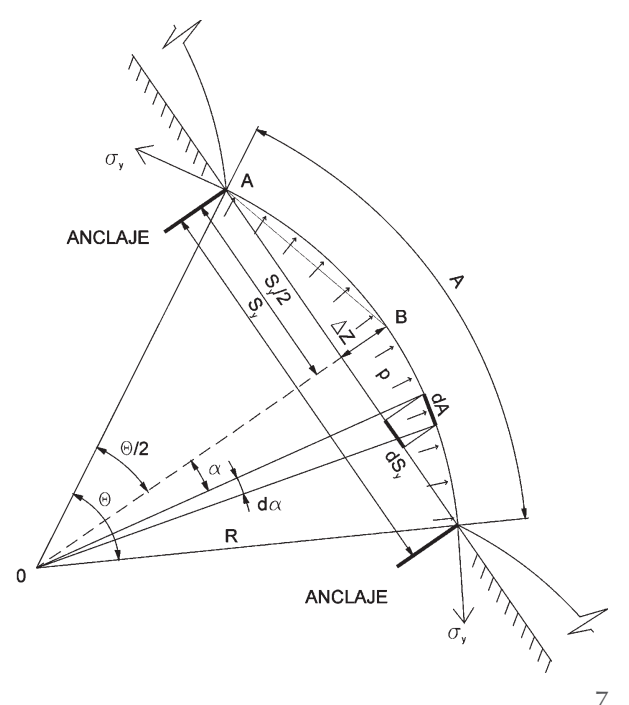

7. Esquema del modelo de deformación plana: sy= carga de trabajo de la malla en la dirección principal; $r=$ presión que ejerce el terreno sobre la malla; $\mathrm{R}=$ radio de curvatura de la malla.

La tensión de trabajo valdrá:

$\sigma_{y}=E_{y c} \varepsilon_{y}$

Donde $\varepsilon_{y}$ se obtiene de la ecuación [5].

Los cálculos realizados con ambos métodos dan prácticamente el mismo resultado lo cual es lógico, ya que se utilizan básicamente las mismas ecuaciones (16).Además se han realizado ensayos en deformación plana, en los cuales se ha sometido una malla horizontal a una presión vertical $(11,12)$. La flecha del arco en rotura obtenida de los ensayos casi coincide con la deducida del cálculo. Respecto a la capacidad de soporte total de la malla en el modelo es superior a la deducida del cálculo, debido a que la forma de la deformada es más bien una parábola que una circunferencia. Como resumen de los ensayos se obtiene la Figura 8, que liga el desplazamiento relativo de la malla con la carga relativa (relación entre la carga que actúa sobre la malla y la carga de rotura), y que corresponde aproximadamente a la ecuación:

$Q / Q r=0,0205\left(\Delta Z / S_{y}\right)^{3}-0,0754\left(\Delta Z / S_{y}\right)^{2}+$
$1,151\left(\Delta Z / S_{y}\right)$
[19] 


\subsection{Cálculo de la malla del Tajo (véase la Figura 6)}

Se va a realizar el cálculo con una presión p de $20 \mathrm{kPa}$.

$S_{y}=2,5 \mathrm{~m} ; S_{x}=5,0 \mathrm{~m}$

Para una malla de $3 \mathrm{~mm}$ de espesor, la ecuación [15] da:

$\Delta Z=0,272 \mathrm{~m}$

De la ecuación [17]:

$\theta(\mathrm{rad})=0,8576$

De la ecuación [9]:

$R=3,01 \mathrm{~m}$

$A=R \theta(\mathrm{rad})=2,58 \mathrm{~m}$

$\varepsilon_{y}=\frac{A-S_{y}}{S_{y}}=\frac{2,58-2,5}{2,5}=0,0313$

$\sigma_{y}=E_{y c} \varepsilon_{y}=59,4 \mathrm{kN} / \mathrm{m}$ admisible

En este caso es:

$\frac{\Delta Z_{y}}{S_{y}}=\frac{0,272}{2,5}=10,9 \%$

$\sigma_{y} / \sigma_{y r}=59,4 / 151,46=39,2 \%$

La Figura 8 nos daría un valor del 30,1\%.

Aplicando un postesado del 15\% de la carga de rotura se elimina la parte inicial de la flecha del arco, correspondiente a Q/Qr=15\%, es decir un $8 \%$ de la deformación relativa (Figura 8). Con ello se obtendría:

$\Delta Z / S_{y}=10,9 \%-8 \%=2,9 \%$

$\Delta Z=0,073 \mathrm{~m}$

Capacidad de soporte de la malla por anclaje [ecuación 14]:

$Q=20 \times 5 \times 2,5=250 \mathrm{kN}$

\section{ACELERACIÓN DE CÁLCULO SEGÚN LA NORMA NCSR-02}

La Norma de Construcción Sismorresistente (NCSR-02) considera como construcciones de especial importancia las construcciones catalogadas como monumentos históricos o artísticos. Dado que la Alhambra es Patrimonio de la Humanidad, parece que el Tajo de San Pedro, que puede hacer peligrar dicho monumento, debe estar en este grupo. Granada tiene una aceleración sísmica básica $a_{b} / g=0,23$ y un coeficiente de contribución (K) de 1.

La aceleración sísmica de cálculo será:

$a_{c}=S \rho a_{b}$, siendo: $\rho=$ coeficiente adimensional de riesgo sísmico, función de la probabilidad de que se exceda en el período de vida, $t$, para el que se proyecta la construcción.

$\rho=1,3$ para construcciones de importancia especial (período de retorno de 1.000 años) $S=$ Coeficiente de amplificación del terreno.

Para $0,1<\rho a_{b}(0,30 \mathrm{~g})<0,4 \mathrm{~g}$ :

$s=\frac{\mathrm{C}}{1,25}+3,23\left(\rho \frac{\mathrm{a}_{\mathrm{b}}}{\mathrm{g}}-0,1\right)\left(1-\frac{\mathrm{C}}{1,25}\right)$

$\mathrm{C}=$ coeficiente del terreno

Para $V_{s}=800$ a $830 \mathrm{~m} / \mathrm{s}$, en terreno tipo I:

$C=1,0$

$s=\frac{1}{1,25}+3,33(1,3 \times 0,23-0,1)\left(1-\frac{1}{1,25}\right)=0,933$

$a_{c}=0,933 \times 1,3 \times 0,23 g=0,28 g$

Si se supone que cada terremoto de aceleración $\geq a_{c}$ que llega a un emplazamiento es un evento en un proceso de Poisson, la probabilidad de que se produzca al menos un evento en el tiempo $t$ es:

$P(x \geq 1, t)=1-e^{-\lambda t}$

Siendo $\lambda$ el número medio de eventos por unidad de tiempo. El valor medio de los intervalos de tiempo entre eventos se llama período de retorno, y vale $1 / \lambda$. El coeficiente $\rho$ que da la Norma Sismorresistente para construcciones de especial importancia corresponde a un período de retorno de 1.000 años. Esto quiere decir que la probabilidad de que se produzca un evento en un período de tiempo de 100 años es del 9,5\% según la ecuación [23]. En cálculo sísmico se admite, con frecuencia, un cuantil de probabilidad que no debe ser excedido del $10 \%$.

\section{ESTABILIDAD DE TALUDES}

Los parámetros de cálculo adoptados para el conglomerado son los que figuran en la Tabla 1 en la columna correspondiente. La elección de estos parámetros se justifica en el apartado 6. Se utilizó en los cálculos el programa Geo-Slope (17), y el método de Morgenstern y Price, con diferentes presiones de malla aplicados a la superficie del talud. El coeficiente de seguridad se define como el valor medio a lo largo de la superficie de deslizamiento del cociente entre la tensión cortante de rotura y la tensión cortante de servicio. Los coeficientes de seguridad estáticos y dinámicos están incluidos en la Tabla 3. No existen grandes diferencias en la superficie de deslizamiento crítica al va- 
riar la presión aplicada por la malla. El incremento de los coeficientes de seguridad estáticos para presiones de malla superiores a 20 kPa es pequeño. Se utilizó en los cálculos dinámicos una aceleración de 0,28 g, según se indica en el apartado anterior. La Figura 9 muestra la superficie de deslizamiento crítica bajo condiciones dinámicas para una presión de $20 \mathrm{kPa}$ sobre el talud. Puede observarse cómo esta superficie crítica penetra dentro de la muralla de La Alhambra.

\section{ANCLAJES}

En primer lugar se perforan y colocan los anclajes del pie del talud, coronación y laterales. Además se deben colocar inicialmente los anclajes en las zonas deprimidas del terreno. Se fijan los cables horizontales y verticales a los anclajes extremos y se tesan. Después de la fijación preliminar de la malla en todo el perímetro y en los puntos deprimidos de la superficie, se perfora el resto de los anclajes.Se aprietan, de modo preliminar, todas las fijaciones de los anclajes interiores. Se aplica la carga de tesado a los anclajes. Los anclajes interiores pueden ser barras Gewi, de acero B-500 S autorroscables, o barras autoperforantes. Estas últimas tienen la ventaja de que no precisan una perforación previa. El diámetro de la barra de anclaje depende de la carga del anclaje, obtenida mediante la [ecuación 14], del límite elástico del acero y del coeficiente de seguridad mínimo adoptado (normalmente 1,67). Los diámetros estándar de las barras Gewi son 25, 28, 32 y $40 \mathrm{~mm}$. La tensión de los anclajes se puede dar mediante llave dinamométrica o gatos hidraúlicos. Al ser muy elevada la altura del Tajo de San Pedro y muy empinado el talud, todos los valores (diámetro de las barras, longitud de anclajes y carga de trabajo de éstos) están en el límite superior de los utilizados en otras obras. El tesado de los anclajes puede requerir la construcción de andamios provisionales, además de colgar al operario de la parte superior del talud. La carga de trabajo de cada anclaje es de 250 kN. La zona estable para el anclaje de los bulones se encuentra por detrás de

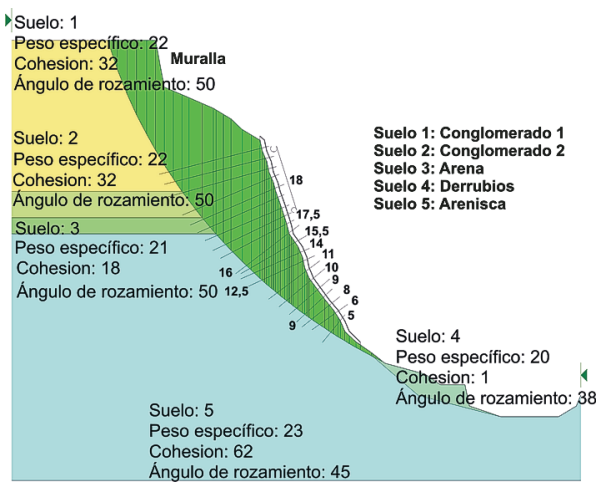

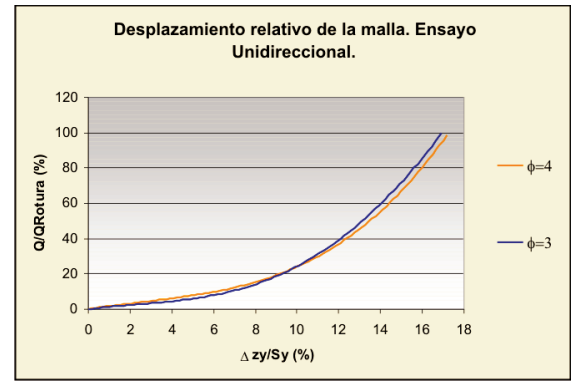

Tabla 3

Coeficientes de seguridad en cálculos de estabilidad estáticos y dinámicos

\begin{tabular}{|c|c|c|}
\hline \multirow{2}{*}{$\begin{array}{c}\text { Presión sobre } \\
\text { el talud }\end{array}$} & \multicolumn{2}{|c|}{$F$} \\
\cline { 2 - 3 } & Estáticos & Dinámicos \\
\hline 0 & 1,132 & 0,73 \\
\hline 10 & 1,31 & \\
\hline 15 & 1,37 & \\
\hline 20 & 1,432 & $\begin{array}{l}(c, 931 \\
(c=32 \mathrm{kPa})\end{array}$ \\
\cline { 3 - 3 } & & $\begin{array}{l}1,002 \\
(c=75 \mathrm{kPa})\end{array}$ \\
\hline 25 & 1,48 & \\
\hline 30 & 1,53 & \\
\hline
\end{tabular}

la superficie de deslizamiento de la Figura 9. De no ser así, el anclaje no evitaría el deslizamiento global, pero sí la caída de lajas superficiales, que es el fenómeno que se ha ido produciendo hasta ahora. Una de las posibilidades es utilizar como anclajes barras tipo GEWI. Con un coeficiente de seguridad de 1,67, la carga en el límite elástico debería ser:

$T_{B R}=250 \times 1,67=417,5 \mathrm{kN}$

La barra tipo GEWI cuya carga en el límite elástico no es inferior a esta cantidad es la de $40 \mathrm{~mm},(628 \mathrm{kN})$.

$$
F=628 / 250=2,5 \text {. }
$$

Se supone un diámetro de perforación de $64 \mathrm{~mm}$. Se supone una adherencia límite entre la lechada y el conglomerado de 0,66 $\mathrm{MPa}$.

Longitud del bulbo en rotura:

$L_{b r}=250 /(660 \times \pi \times 0,064)=1,884 \mathrm{~m}$

Longitud del bulbo en condiciones de trabajo, con un coeficiente de seguridad de 1,65 por ser anclaje permanente (18):

$L_{b}=1,884 \times 1,65=3,1 \mathrm{~m}$
8. Relación entre el desplazamiento relativo de la malla y la carga relativa (cortesía de Geobrugg).

9. Superficie de deslizamiento crítica, bajo condiciones dinámicas, que penetra en los muros de La Alhambra. 
El bulbo de anclaje debería estar situado detrás de la superficie de deslizamiento (Figura 9).

\section{IMPACTO AMBIENTAL}

Se ha hecho un estudio de las especies protegidas y se ha visto que la obra apenas les afecta. La malla se va a colorear para mimetizarla con el terreno. Se ha hecho un estudio del impacto visual desde distintas cuencas mediante simulaciones en ordenador $y$, debido a la mimetización, al pequeño diámetro y a la amplia abertura de la malla, se ha llegado a la conclusión de que dicho impacto es perfectamente asumible.

\section{CONCLUSIONES}

El Tajo de San Pedro es un abrupto diedro de $65,5 \mathrm{~m}$ de altura, cuya proximidad a la muralla-palacio de La Alhambra, que es patrimonio de la Humanidad, supone un peligro para ésta. Los desprendimientos de tierras y los desplazamientos de algunas faIlas normales han destruido ya una parte importante de la valla cristiana, construida en
1526. El Tajo está rodeado por fallas activas normales. Una de las caras del diedro es un escarpe de línea de falla (fault-line scarp), ya que el escarpe de falla original se ha visto sometida a procesos de erosión posteriores (19). Se ha creado un régimen tectónico de extensión que afloja el terreno y activa su caída. El coeficiente de seguridad del Tajo sometido al terremoto de periodo de retorno 1.000 años es 0,73 . Tras analizar otras posibles soluciones, se ha propuesto para su preservación una malla de alambre de alto límite elástico con anclajes postesados y vegetación autóctona. Se incluyen en este artículo los cálculos de la malla y de los anclajes. Con este refuerzo, el coeficiente de seguridad bajo el terremoto de proyecto se eleva a un valor en torno a uno. Con esta solución el impacto medioambiental es insignificante.

\section{RECONOCIMIENTOS}

Este trabajo se ha realizado con ayuda del proyecto de investigación BIA 2004-01302 del Ministerio de Educación y Ciencia.

\section{BIBLIOGRAFÍA}

(1) Rodríguez Ortiz, J. Ma, 1998. "El Tajo de San Pedro de La Alhambra de Granada". Inf. no publicado.

(2) Cedex, 1993. "Caracterización geotécnica del conjunto monumental de La Alhambra y Generalife". Informes parciales $n^{\text {os }} 1,2$ y 3.

(3) Geocisa, 2002. "Reconocimientos geotécnicos del tajo de San Pedro, Granada". Anejo n 3 del 'Proyecto de Rehabilitación del Tajo de San Pedro de La Alhambra de Granada'.

(4) Justo, J.L., Vázquez, N. y Durand, P., 2005. "Proyecto de restauración del Tajo de San Pedro de La Alhambra de Granada". Conf. Hidrográfica del Guadalquivir y Patronato de La Alhambra y Generalife.

(5) Jiménez Salas, J. A. y Uriel, S., 1964. "Some recent Rock Mechanics Testing in Spain". $8^{\circ}$ Cong. de Grandes Presas, Edimburgo, 995-1021.

(6) Centre d'Etudes Ménard, 1970. "Détermination de la Poussée Exercée par un Sol sur une Paroi de Soutènement", Publication D/60/75.

(7) Baguelin, F., Jézéquel, D. H. y Shields, D. H., 1978. "The Pressuremeter and Foundation Engineering". Trans Tech Publications, Aedermansdorf, Suiza.

(8) Torres Vila, J. A. 1999. "Cálculo y diseño de sostenimientos mediante red de cables anclada. Sistema Pentifix". Jornada Técnica Soluciones Flexibles en el Tratamiento de Taludes, Granada.

(9) Muñoz, B., y Torres Vila, J.A. 2000. "Sistemas de soporte flexibles en la estabilización de taludes y control de la erosión. Experiencias de aplicación en Andalucía". Segundo Congreso Andaluz de Carreteras, Cádiz, 2, 1349-1362.

(10) Geobrugg. TECCO, tecnología de estabilización y control de la erosión de taludes y laderas inestables.

(11) Torres Vila, J.A., Torres Vila, M.A. y Castro, D. 2001b. "Caracterización mecánica de la membrana de alambre de acero de alto límite elástico Tecco G-65, como elementos de soporte superficial en la estabilización de taludes". IV Simposio Taludes y Laderas Inestables, Madrid, 3: 1157-1166.

(12) Castro, D. y Ballester, F., 2003. "Sistema flexible unidireccional para la estabilización de taludes y laderas de suelos o materiales sueltos". Ingeniería Civil, 129: 41-50.

(13) He, Yong-Kui, 2006. "Concepts and methods of slope flexible stabilisation system". Chinese Journal of Rock mechanics and Engineering, 25: 2: 217-225. En chino.

(14) He, Yong-Mei, 2006. "Typical cases of slope protection system". Chinese Journal of Rock mechanics and Engineering, 25: 2: 323-328. En chino.

(15) Torres Vila, J.A., Torres Vila, M.A. y Castro, D. 2001a. "Validación de los modelos físicos de análisis y diseño para el empleo de membranas flexibles Tecco G-65, como elemento de soporte superficial en la estabilidad de taludes". IV Simposio Taludes y Laderas Inestables, Madrid, 3: 1107-1118.

(16) Castro, D., Torres Vila, J. A. y Torres Vila, M. A., 2001. "Modelos físico-matemáticos de análisis y diseño para el empleo de membranas flexibles como elementos de soporte en la estabilización superficial de taludes". IV Simposio Taludes y Laderas Inestables, Madrid, 3: 1017-1028.

(17) Geo-Slope Office, 1998. "Slope/W for slope stability analysis. Version 4". Geo-Slope International Ltd., Calgary, Alberta, Canadá.

(18) Ministerio de Fomento, 2004. "Guía para el diseño y la ejecución de anclajes al terreno en obras de carretera". Ministerio de Fomento.

(19) Justo, J.L., Azañón, J.M., Azor, A., Saura, J., Durand, P., Villalobos, M., Morales A., y Justo , E., 2008. "Neotectonics and slope stabilisationn at the Alhambra, Granada, Spain, 